\title{
18F-fluciclovine (Axumin) PET/CT detecting occult bone metastasis
}

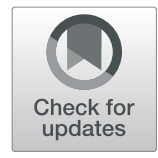

\author{
Pankaj Nepal', Paul Rodrigue and Thomas Olsavsky
}

\begin{abstract}
Background: 18F-fluciclovine (Axumin) positron emission tomography (PET) is a novel agent for detection of biochemical recurrence of the prostate cancer. In this article, we present an interesting case of prostate cancer recurrence with bone metastasis not visualized with conventional $C T$, but detected with Axumin PET/CT. This case report will help reader to understand shortcomings of conventional modalities (CT/MRI/bone scan) in evaluation of biochemical recurrence of prostate cancer.

Case presentation: We report a case of 73-year-old male previously diagnosed with adenocarcinoma of prostate, status post-radiation treatment with excellent response to therapy. He presented with progressively increasing right groin pain and rising PSA level indicating biochemical recurrence. A bony metastasis to right inferior pubic ramus was diagnosed with Axumin PET/CT, not detected with conventional CT. He underwent local radiation treatment with excellent response to therapy.

Conclusion: 18F-fluciclovine (Axumin) PET/CT is the most sensitive tool in detection of biochemical recurrence of prostate cancer. CT and MRI may not detect early cases of tumor recurrence which affects disease prognosis and patient management.
\end{abstract}

\section{Background}

About $1 / 3^{\text {rd }}$ cases of prostate cancer develop biochemical recurrence during post-treatment surveillance. After treatment for localized prostate cancer, patients are followed up with serial PSA level to detect biochemical recurrence [1]. 18F-fluciclovine (Axumin) is a novel radiolabeled amino acid analog with higher uptake in prostate cancer cells than in the surrounding tissues. Although 18Ffluorodeoxyglucose (18F-FDG) is a widely available workhorse tracer agent for vast majority of the cancers, it has limited role in prostate cancer imaging due to its poor sensitivity for both initial staging as well as recurrence localization. Poor sensitivity is attributed to most prostate cancers utilizing non-glucose metabolic pathway, small size of recurrent lesions, and adjacent intense urinary bladder activity [1, 2]. 18F-fluciclovine (Axumin) is the only ${ }^{18} \mathrm{~F}$-based radiotracer that the US Food and Drug

\footnotetext{
* Correspondence: Pankaj-123@live.com

Department of Radiology, St. Vincent's Medical Center, Bridgeport, Connecticut 06606, USA
}

Administration (FDA) has approved for molecular imaging in patients suspected of having recurrent prostate cancer. It has better recurrence detection rate than $11 \mathrm{C}$ choline and 18F-FDG positron emission tomography (PET); currently, it is the most sensitive approved novel agent for detection of biochemical recurrence of the prostate cancer [2]. Axumin PET is indicated in localizing recurrent disease in prostate/prostatectomy bed, pelvic or distant lymph nodal disease, and bony as well as distant/ visceral metastasis in patients with rising PSA after treatment completion [1,2]. Detecting sites of relapse and characterizing extent of tumor provide crucial information for appropriate treatment planning. We present an interesting case where the bony metastasis was not seen with CT pelvis but detected with Axumin PET/CT highlighting the role of novel PET tracer to detect biochemical recurrence of prostate cancer.

\section{Case presentation}

Four years back, a 73-year-old male was diagnosed with adenocarcinoma of the prostate with Gleason score 6 .

\section{Springer Open}

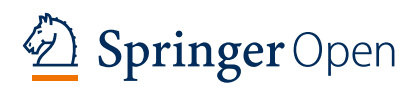

(c) The Author(s). 2020 Open Access This article is licensed under a Creative Commons Attribution 4.0 International License, which permits use, sharing, adaptation, distribution and reproduction in any medium or format, as long as you give appropriate credit to the original author(s) and the source, provide a link to the Creative Commons licence, and indicate if changes were made. The images or other third party material in this article are included in the article's Creative Commons licence, unless indicated otherwise in a credit line to the material. If material is not included in the article's Creative Commons licence and your intended use is not permitted by statutory regulation or exceeds the permitted use, you will need to obtain permission directly from the copyright holder. To view a copy of this licence, visit http://creativecommons.org/licenses/by/4.0/. 
The patient then underwent radiation treatment with excellent response to therapy and undetectable PSA. He presented to his physician with progressively increasing right groin pain for a month, 4 years after the treatment completion. On physical examination, there was deep right groin tenderness, but no mass was palpable. Rest of the physical examination was unremarkable. A noncontrast CT of pelvis did not show significant abnormality apart from focal anterior cortical bulge (Fig. 1). His PSA level was markedly elevated $(6.1 \mathrm{ng} / \mathrm{mL})$ from his baseline $(<0.1 \mathrm{ng} / \mathrm{mL})$ indicating biochemical recurrence of the prostate cancer. Only $<5 \%$ of bone scans are positive for detecting bony metastasis at PSA level $<7 \mathrm{ng} /$ $\mathrm{mL}$, as well as bone scans are more sensitive to detect sclerotic lesions [1]. Since our patient had radiologically occult disease with no significant abnormality on pelvic CT, our multidisciplinary team thought to proceed with Axumin PET/CT. Moreover, we were not sure whether our patient had radiologically occult bony/soft tissue lesions, lymph nodal, or distant metastasis. After insurance approval for reimbursement, Axumin PET/CT scan was performed which showed increased tracer activity in right inferior pubic ramus with standardized uptake value maximum (SUVmax) 7.4 (Figs. 2 and 3) consistent with bony metastasis. CT guided biopsy of the lesion revealed metastatic adenocarcinoma of the prostate. Patient was again treated with local radiation therapy and currently under surveillance with nadir PSA $<0.1 \mathrm{ng} /$ $\mathrm{mL}$. His symptoms of right groin pain also subsided.

\section{Discussion}

Food and Drug Administration (FDA) has approved 18F-fluciclovine (Axumin) for workup of the prostate cancer biochemical recurrence. Radiology and nuclear

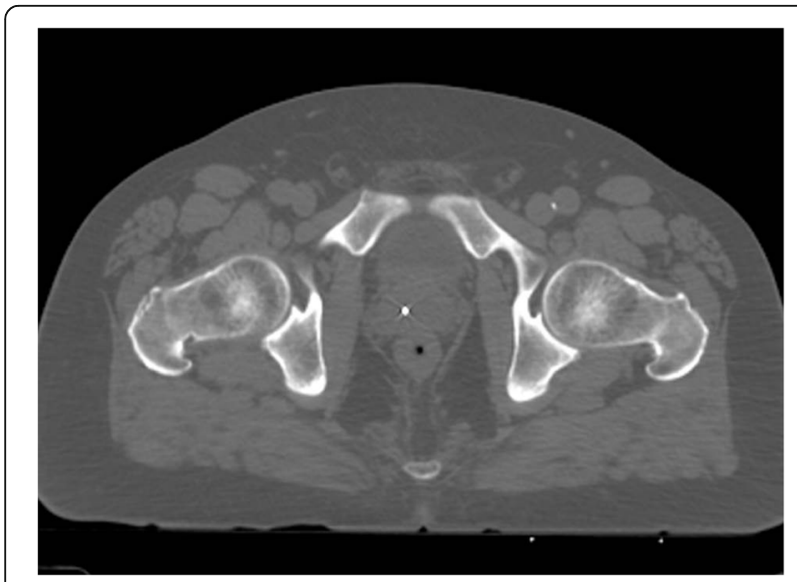

Fig. 1 Non-contrast CT of pelvis axial image did not show definite bony metastasis, but abnormal focal cortical bulge, which was inconclusive. Note is made of permanent radioactive seeds within the prostate from prior treatment medicine residents, clinicians, and reporting radiologists should be aware of the characteristics, imaging protocol, and interpretation criteria for Axumin PET/CT. 18Ffluciclovine (Axumin) is a leucine amino acid analog which can move across the tumor cells fairly rapidly, with peak time ranging 4 to $10 \mathrm{~min}[3,4]$. Images can be obtained 3 to 5 min post-injection, quickly compared to fluorodeoxyglucose (FDG) PET/CT. Patient must fast for at least $4 \mathrm{~h}$ to reduce amino acid level in the blood which can interfere competing with the tracer [4-6]. Contrary to FDG-PET/CT, Axumin PET/CT is performed with full bladder because of very minimal renal excretion and urinary bladder activity [5]. This allows for better evaluation of prostate bed. Intense tracer uptake is seen in liver and pancreas [6]. Variable uptake is noted in salivary glands, bone marrow, and muscles. Unlike FDG, uptake in brain is minimal [6].

The most intense physiologic tracer uptake is seen in the pancreas, which decreases within $15 \mathrm{~min}$ after injection to a level lower than that of the liver. The liver has second most intense physiologic activity and is the most critical organ for radiation [1,6]. Axumin PET image interpretation is predominantly visual or qualitative, rather than measurement of actual SUV. Radiotracer uptake in suspicious lesions is compared with uptake in the bone marrow or blood pool. Bone marrow in third lumbar vertebra (L3) is recommended as a standard comparator because it is less likely to have arthritic changes that could confound interpretation of tracer uptake $[2,6]$. Tracer uptake equal to or greater than that of the blood pool but less than that of the bone marrow is considered to be mild. Tracer uptake equal to or greater than that of bone marrow but less than that of the liver is considered to be moderate, and avidity equal to or greater than that of the liver is considered to be intense [1]. Lytic bone metastases typically show intense uptake, followed by mixed (lytic and sclerotic) bone lesions which commonly show moderate uptake. It should be noted that sclerotic metastatic lesions may have no tracer uptake with Axumin PET. Being an 18F radiotracer, an on-site cyclotron is not required for its production, which enables widespread clinical availability of this agent [1]. Compared to 18F-FDG, however the cost is high [6].

Axumin PET findings can alter treatment modification by finding otherwise undetected metastasis. After definitive treatment for prostate cancer, patients are routinely followed up with serum PSA levels. Imaging with CT and MRI may not detect or accurately characterize the biochemical relapse at earliest such as in our case. Multiple imaging modalities are available; usually imaging performed with computed tomography (CT), magnetic resonance imaging (MRI), and bone scan to evaluate 

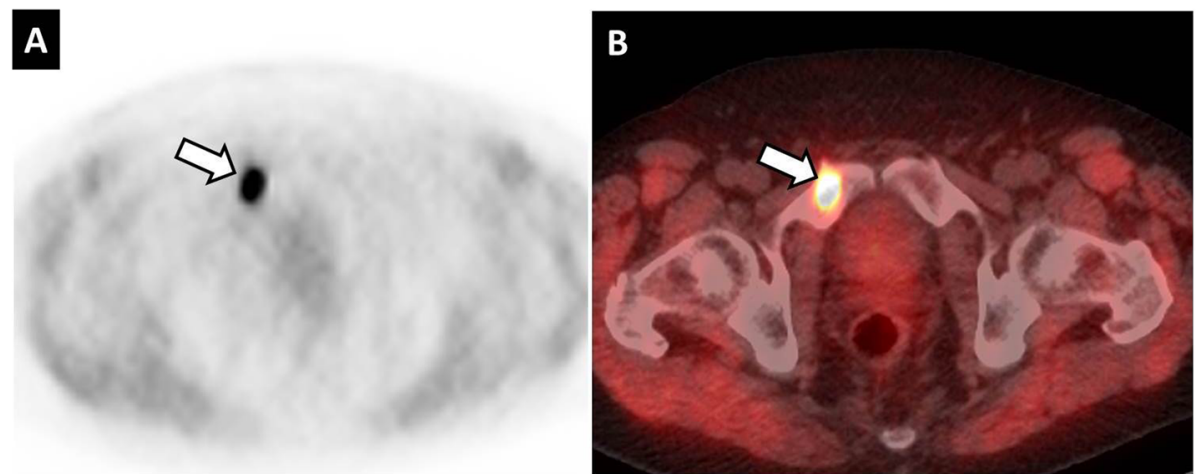

Fig. 2 a Axial 18F-fluciclovine (Axumin) PET and b PET/CT fusion images showing increased activity in right inferior pubic ramus (arrow) with standardized uptake value maximum (SUVmax) 7.4 consistent with recurrent bony metastasis

prostate cancer recurrence. CT and MRI provide anatomic information and detect the mass when it is obvious, but do not provide physiological information. Also, it may be difficult to detect local tumor recurrence at prostate bed due to radioactive seeds. Metastasis to bone and lymph nodes may not be detected early with conventional imaging [6]. Bone scan has low sensitivity and specificity in detecting bony metastasis, and further it cannot evaluate lymph nodal or distant metastasis. Axumin PET is superior to bone scan because bone scan only detects osteoblastic process, whereas Axumin PET shows both bony and soft tissue tumors.

Axumin PET has detection rates of $72.0 \%, 83.3 \%$, and $100 \%$ at PSA levels $<1,1-2$, and equal or more than 2 $\mathrm{ng} / \mathrm{mL}$, respectively [7]. It has negative predictive value (NPV) of $100 \%$ with PSA value of more than $1.05 \mathrm{ng} /$ $\mathrm{mL}$. The findings on Axumin PET may be crucial in treatment modification such as widening radiation field when more local lesions are seen or avoiding radiation altogether because distant metastasis is seen, not identified by other structural imaging modalities such as CT/ MRI or other PET tracers. Axumin uptake can be detected in benign prostatic hypertrophy nodules in the residual prostate tissue and it is still not indicated for evaluation of initial prostate cancer staging $[1,2]$. Axumin PET is in initial phase; thus, further research and case studies will definitely enrich us with knowledge about this novel PET tracer.

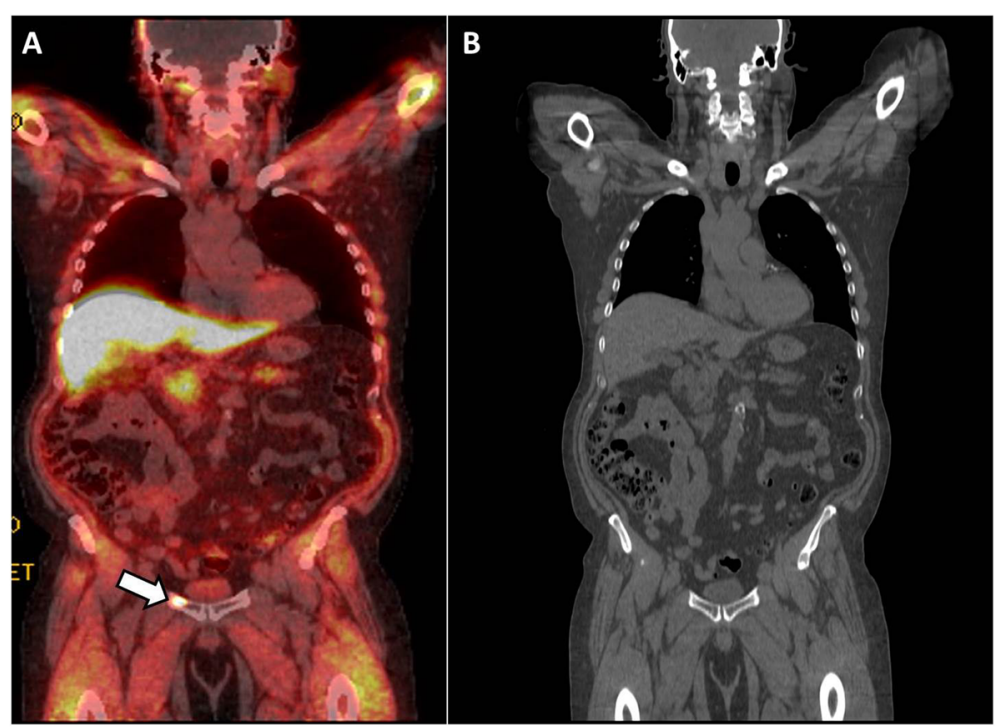

Fig. 3 Coronal 18F-fluciclovine (Axumin) PET/CT fusion image showing increased activity in right inferior pubic ramus (arrow) with standardized uptake value maximum (SUVmax) 7.4 consistent with recurrent bony metastasis. Also note the physiological distribution of 18F-fluciclovine tracer within the liver, pancreas, and visualized muscles. Contrary to FDG PET, brain activity is minimal. b Non-contrast CT image of same patient for comparison 


\section{Conclusion}

18F-fluciclovine (Axumin) PET/CT provides physiological information and most sensitive tool in detection of biochemical recurrence of prostate cancer. Conventional imaging modalities may not detect early cases of tumor recurrence which affects disease prognosis and patient management.

\section{Abbreviations}

PET: Positron emission tomography; FDG: Fluorodeoxyglucose; 18F-FDG: 18Ffluorodeoxyglucose; FDA: Food and Drug Administration; CT: Computed tomography; MRI: Magnetic resonance imaging; PSA: Prostate specific antigen; SUV: Standardized uptake value; NPV: Negative predictive value

\section{Acknowledgements}

We acknowledge our patient, great source of learning.

\section{Authors' contributions}

PN- manuscript writing, PR- manuscript edit, and TO- attending radiologist, data design. All authors have read and approved the manuscript.

\section{Funding}

No one was paid during this study. The study did not have a source of funding. This study was not supported by a grant.

\section{Availability of data and materials}

All data is available based on a reasonable request.

\section{Ethics approval and consent to participate}

The institutional review board (IRB) waiver of informed consent applicable. This case report was a reporting of a case in a medical educational center, in which all patients are informed that they may be subjects of scientific experiments and are informed of the ethical codes of conducts. This study was in compliance to the latest version of the Helsinki Declaration.

\section{Consent for publication}

The institutional review board (IRB) waiver of informed consent is applicable.

\section{Competing interests}

The authors declare that they have no competing interests.

Received: 25 June 2020 Accepted: 24 July 2020

Published online: 30 July 2020

\section{References}

1. Gusman M, Aminsharifi JA, Peacock JG, Anderson SB, Clemenshaw MN, Banks KP et al (2019) Review of 18F-fluciclovine PET for detection of recurrent prostate cancer. Radiographics 39:822-841

2. Fraum TJ, Ludwig DR, Kim EH, Schroeder P, Hope TA, Ippolito JE (2018) Prostate cancer PET tracers: essentials for the urologist. Can J Urol 25:9371-9383

3. Oka S, Kanagawa M, Doi Y, Schuster DM, Goodman MM, Yoshimura H et al (2017) Fasting enhances the contrast of bone metastatic lesions in $18 \mathrm{~F}$ fluciclovine-PET: preclinical study using a rat model of mixed osteolytic/ osteoblastic bone metastases. Int J Mol Sci 18:E934

4. Savir-Baruch B, Zanoni L, Schuster DM (2017) Imaging of prostate cancer using fluciclovine. PET Clin 12:145-157

5. Wallitt KL, Khan SR, Dubash S, Tam HH, Khan S, Barwick TD et al (2017) Clinical PET imaging in prostate cancer. Radiographics 37:1512-1536

6. Songmen S, Nepal P, Olsavsky T, Sapire J (2019) Axumin positron emission tomography: novel agent for prostate cancer biochemical recurrence. J Clin Imaging Sci 9:49

7. Akin-Akintayo OO, Jani AB, Odewole O, Tade Fl, Nieh PT, Master VA et al (2017) Change in salvage radiotherapy management based on guidance with FACBC (fluciclovine) PET/CT in postprostatectomy recurrent prostate cancer. Clin Nucl Med 42:e22-e28

\section{Publisher's Note}

Springer Nature remains neutral with regard to jurisdictional claims in published maps and institutional affiliations.

\section{Submit your manuscript to a SpringerOpen ${ }^{\circ}$ journal and benefit from:}

- Convenient online submission

- Rigorous peer review

- Open access: articles freely available online

High visibility within the field

- Retaining the copyright to your article 